\title{
Potential Effects of Heart Symbol Compliant Foods on Nutrient Intake
}

\author{
${ }^{*}$ Raulio $S^{1}$, Ali-Kovero $\mathrm{K}^{1}$, Tapanainen $\mathbf{H}^{1}$, Toivola $\mathrm{L}^{2}$, Virtanen SM ${ }^{1,3,}$ Lahti-Koski $\mathbf{M}^{2}$ \\ ${ }^{1}$ Nutrition Unit, Department of Health, National Institute for Health and Welfare, Helsinki, Finland
}

${ }^{2}$ Finnish Heart Association, Helsinki, Finland

${ }^{3}$ School of Health Sciences, University of Tampere; Center for Child Health Research, The Science Center of Pirkanmaa Hospital District, Tampere, Finland

Received: February 03,2017; Accepted: March 14,2017; Published: March 21, 2017

*Corresponding author: Susanna Raulio, National Institute for Health and Welfare (THL), P.O. Box 30, FI-00271 Helsinki, Finland Tel. +358 29 524 8574; E-mail: susanna.raulio@thl.fi

\begin{abstract}
Objectives: The aim of this study was to evaluate the potential effect on fat, saturated fat, sodium and sugar intake of revising food compositions according to the Heart Symbol criteria in the Finnish working aged population.

Design: A scenarios of possible food intake was formed and compared with the baseline. Nutrient intake in the baseline was based on the National FINDIET 2012 Survey.
\end{abstract}

Setting: A representative random sample of five regions in Finland.

Subjects: Dietary intake of participants aged 25 to 64 years $(n=1295)$ were gathered using 48-hour recalls for consecutive days.

Results: The results showed significant reductions in fat, saturated fat and sodium. Reductions were parallel for both sexes and took place in all age and educational groups. The mean intake of saturated fat decreased from $14.3 \mathrm{E} \%$ to less than $10 \mathrm{E} \%$. The mean daily intake of sodium decreased by more than $10 \%$.

Conclusion: The study suggests that there is great potential to reduce the intake of saturated fat and sodium by using products bearing the Heart Symbol, with more success in meeting nutrition recommendations.

Keywords: Nutrition Labels; Heart Symbol; Dietary Modelling; Salt; Saturated Fat; Sugar;

\section{Summary}

The aim of this study was to evaluate the potential effect on fat, saturated fat, sodium and sugar intake of revising food compositions according to the Heart Symbol criteria in the Finnish working aged population. A scenario of possible food intake was formed and compared with the baseline. Nutrient intake in the baseline was based on the National FINDIET 2012 Survey. Dietary intake of participants aged 25 to 64 years $(n=1295)$ were gathered using 48-hour recalls for consecutive days. The results showed significant reductions in fat, saturated fat and sodium. Reductions were parallel for both sexes and took place in all age and educational groups. The mean intake of saturated fat decreased from $14.3 \mathrm{E} \%$ to less than $10 \mathrm{E} \%$. The mean daily intake of sodium decreased by more than $10 \%$. The study suggests that there is great potential to reduce the intake of saturated fat and sodium by using products bearing the Heart Symbol, with more success in meeting nutrition recommendations.

\section{Introduction}

Diets high in saturated fatty acids, refined carbohydrates and sodium, but low in fiber are associated with a higher risk of diet-related chronic diseases [1]. To reduce the burden of chronic diseases, the World Health Organization (WHO) has recommended that the food industry should limit the content of saturated and trans fatty acids, sodium, and sugar in existing food products. In addition, developing new products with better nutritional value is recommended [2].

Food manufacturers can offer healthier products and simplify consumers' better choices by using labels that point out clear information about the food characteristics. Front-ofpackage labels are used in many countries to indicate products with healthier nutrient content compared with other products within the same product category. In addition to the facilitation of healthy consumer choices, health logos motivate food manufacturers to reformulate existing products and develop healthier products $[3,4,5]$.

Earlier studies have evaluated the effects of replacing foods on the intake of saturated fatty acids, sugar and sodium [6], saturated fat [7], or saturated fat, total sugar and fiber [8]. These studies showed that diets containing foods complying with the health logo criteria shifted the nutrient intake closer to meet the recommendations. Also a Brazilian study showed that replacing typical products by products complying with the Choices criteria reduced the intake of saturated fat by $52 \%$ and trans fatty acids by $92 \%$ in the Brazilian population. Sodium intake was reduced 
by $47 \%$, whereas fiber intake was increased by $87 \%$ [9].

In Finland, a variety of food products have been labeled with a front-of-package label called the Heart Symbol since year 2000, when the system was launched by the Finnish Heart Association (FHA) and the Finnish Diabetes Association (FDA) [10]. The Heart Symbol is a summary indicator that enables consumers to make healthier choices at a glance as to whether the product contains less fat, saturated fatty acids, and sodium than other products within the same product category. Sugar, fiber and cholesterol content are also acknowledged in some product groups. The Heart Symbol can be set for nine main food groups (milk and dairy products, oils and fats, fish, meat, meat products, bread and cereal products, convenience foods, spices and seasoning sauces, vegetables, fruits and berries), which can be further divided into subgroups. The criteria are defined for each subgroup separately, according to what is relevant to nutrient content and health in that particular food group. In developing the criteria, the Finnish nutrition recommendations [11], the possibilities of food technology, and the properties of food products currently available [12], were taken into account.

According to the National FINDIET 2012 Survey the average intake of saturated fat is still above the recommended $10 \%$ of energy in the Finnish adult population [13]. Also the mean salt intake is above the recommended 5 g per day [13]. The average daily intake of dietary fiber, on the other hand, fall below the recommended 25-35 grams a day in working-aged adults. The main sources of saturated fat are milk and dairy products, meat products and bakery products in the Finnish diet. The main contributors to salt intake are cereal products, while together with meat products and meat dishes, they account for over $60 \%$ of the intake. Rye and mixed flour breads are the foremost contributors to fiber intake [13].

Excessive intake of saturated fat and sodium has been subject for many prevention programs in Finland for decades, since they are associated with increased serum cholesterol level and elevated blood pressure - both major contributors for coronary heart disease [14]. As was recently pointed out with Jousilahti et al. [14] primary prevention and reduction in levels of the main factors contributing to cardiovascular risk, including intake of saturated fat and salt, should still be considered as the main strategy to reduce disease burden and mortality due to coronary heart disease.

We hypothesize that the majority of the most important weaknesses in the diet of Finnish adult population mentioned above could be improved by replacing regular food products by foods meeting the criteria of the Heart Symbol. The aim of this study was to calculate the intake of fat, saturated fat, sodium, sugar and fiber after revising computationally food compositions of some most important food groups according to the Heart Symbol criteria.

\section{Material And Methods \\ Food consumption data}

The dietary data were derived from the National FINDIET 2012 Survey [13]. The FINDIET 2012 Survey was conducted between January and April 2012 as part of The National FINRISK Study [15], which monitors risk factors for chronic, non-communicable diseases. A random sample of adults aged 25-74, stratified by sex, region and 10-year age-groups was drawn from the population register of five regions of Finland. Onethird of the participants of the FINRISK 2012 Study were asked to participate in a 48-h dietary recall [13]. In the present study, we focused on working-aged adults aged 25-64 years old $(n=1295)$ who completed the dietary interview. The participation rate was $51 \%$ of those invited to participate in the dietary survey. Dietary data were collected using a computer-assisted 48-h dietary recall that was carried out by trained nutritionists [16]. A more detailed description of the dietary data collection is given by [17].

\section{Scenario for nutrient dietary modelling}

We created a scenario, in which products in the defined food groups not complying with the Heart Symbol criteria were replaced by similar foods complying with the criteria. The average nutritional content of products labeled with the Heart Symbol (products on the Finnish market in December 2014) were used.

Replacements were performed in four main food groups: milk and dairy products (milk, buttermilk, vegetable milks, cream, yoghurt, fermented milk, curd, pudding, soft cheese, cheese spread, hard cheese), meat products (cold cuts, sausages), fats and oils (fat spreads) and bread and cereal products (bread, biscuit and pastry items). These four food groups were selected since they belong to the main sources of saturated fat, salt and fiber, and thus were assumed to have great potential in reducing saturated fat and salt intake and increasing fiber intake in the Finnish adult population. Only foods used as such were replaced, whereas ingredients in recipes were not changed. The amount of food items consumed was kept the same as original foods in the baseline scenario.

\section{Replacement procedure}

The composition data of replacement foods were added to the composition database by creating new food codes for 'Heart Symbol' products. The nutrient content was changed by revising the product composition only in relation with the nutrient in question. The aim was to create a product that resembled the original product as much as possible. For instance, the criteria for fat spreads in the Heart Symbol takes into account the type of fat but the amount of fat is not regulated. Therefore, the total fat content was kept as close to the original product as possible. In total, 99 new codes (products) were added to the database. 
The replacements were made only for foods that were used as such, not for foods included in recipes. Whole milk, for example, was replaced when drank with the porridge, but if the porridge was cooked with milk, it was not replaced.

Term "sugar" in the nutrition facts table and the sugar criterion of the Heart Symbol indicates total sugar, which refers to all mono- and disaccharides naturally occurring, or added to foods. To calculate the sucrose content in the new codes, the average nutrient content of foods labeled with the Heart Symbol was used and the relation of sucrose to total sugar was maintained.

The saturated fatty acid criterion of the Heart Symbol signifies "hard fat", that is, saturated fatty acids and trans fatty acids combined.

\section{Food composition database}

Nutrient intake was calculated with in-house software built using data from the Finnish Food Composition Database Fineli ${ }^{\circledR}$ [18]. After identifying foods belonging to the defined food groups they were evaluated against the Heart Symbol criteria and allocated into compliant and non-compliant. Compliant foods met all of the criteria set for the corresponding food group. The criteria are based on the amount and type of fat, the amount of sodium and in some groups, also sugar, fiber and cholesterol. The classification resulted in 108 products that complied with the label's criteria and 665 non-compliant products $(23 \%$ of the 2928 different food items).

\section{Statistical analyses}

The mean intake of nutrients was calculated for the baseline and the scenario by sex, 10-year age group and education. Education (in years) was categorized into tertiles according to sex and birth year. The differences in nutrient intake between the baseline and the scenario were calculated and the differences were tested using a paired t-test or the Wilcoxon signed rank test, depending on the distribution of the difference. To analyze whether the difference between the baseline and the scenario was equal across all age groups or education groups, mixed models for repeated measurements were constructed with the interaction term of the measurement (baseline/scenario) and age group or education group. Logarithmic or square root transformations were used to improve the normality of repeated intake measures. A p-value of $<0.05$ was considered statistically significant. The analyses were done using the SAS statistical package (SAS Institute Inc., Cary, NC, USA, version 9.3).

\section{Results}

\section{Intake assessment}

Nutrient intake was estimated at baseline (eg. the original dietary data) and in the scenario (Table 1). Fat, hard fat, sodium, sucrose and energy showed significant reductions for the scenario, whereas the intake of fiber increased. Differences were similar for both sexes.

Table 1: Mean nutrient intake at baseline and in the scenario, by sex.

\begin{tabular}{|c|c|c|c|c|c|c|}
\hline & \multicolumn{2}{|c|}{ Men, $n=585$} & \multicolumn{2}{|c|}{ Women, $n=710$} & \multicolumn{2}{|c|}{ Total, $n=1295$} \\
\hline & Baseline & Scenario & Baseline & Scenario & Baseline & Scenario \\
\hline Energy (kJ) & 9386.1 & 8835.6 & 7253.4 & 6871 & 8216.8 & 7758.5 \\
\hline Fat (g) & 92.5 & 77.5 & 70.5 & 59.5 & 80.4 & 67.6 \\
\hline $\begin{array}{c}\text { Hard fats (SFA + trans) } \\
\text { (g) }\end{array}$ & 37.2 & 24 & 28.3 & 18.8 & 32.3 & 21.1 \\
\hline Protein (g) & 94.5 & 96.4 & 72 & 73.8 & 82.2 & 84 \\
\hline Carbohydrate (g) & 230.4 & 232.5 & 185.8 & 188 & 205.9 & 208.1 \\
\hline Sucrose (g) & 51.2 & 47.2 & 43.7 & 39.6 & 47.1 & 43 \\
\hline Sugar (g) & 107.8 & 103.2 & 92 & 87.8 & 99.2 & 94.7 \\
\hline Fiber (g) & 22 & 23 & 20.5 & 21.2 & 21.2 & 22 \\
\hline Sodium (g) & 3.5 & 3.1 & 2.6 & 2.3 & 3 & 2.7 \\
\hline Salt (g) & 8.9 & 7.9 & 6.5 & 5.8 & 7.6 & 6.8 \\
\hline Fat (\% energy) & 36.1 & 32.2 & 35.5 & 31.7 & 35.8 & 31.9 \\
\hline $\begin{array}{c}\text { Hard fats (SFA + trans) } \\
(\% \text { energy })\end{array}$ & 14.4 & 9.9 & 14.2 & 10 & 14.3 & 9.9 \\
\hline Protein (\% energy) & 17.3 & 18.7 & 17.2 & 18.6 & 17.2 & 18.6 \\
\hline Carbohydrate (\% energy) & 42 & 44.9 & 43.7 & 46.6 & 43 & 45.8 \\
\hline Sucrose (\% energy) & 9.2 & 9 & 10.1 & 9.6 & 9.7 & $9.3^{*}$ \\
\hline Sugar (\% energy) & 19.8 & 20.1 & 21.7 & 21.8 & 20.8 & 21 \\
\hline Fiber (g/M) & 2.4 & 2.7 & 2.9 & 3.2 & 2.7 & 2.9 \\
\hline
\end{tabular}


The average intake of hard fat was $32.3 \mathrm{~g}$ per day or $14.3 \mathrm{E} \%$ in the baseline, which is well above the recommended $10 \%$ of energy. After replacing non-compliant foods with the Heart-Symbol compliant foods, the intake of hard fat met the recommendations in the scenario (9.9 E\%). Hard fat intake was reduced by $34.6 \%$ from baseline in the scenario (Table 1). Decrease in total fat intake was smaller, from $80.4 \mathrm{~g}$ per day (35.8 E\%) to $67.6 \mathrm{~g}$ a day (31.9 E\%) in the scenario (Table 1). In percentages, the reduction was $15.9 \%$.

The average daily intake of salt was 7.6 grams at baseline but after the replacements it was reduced by $11.0 \%$ to $6.8 \mathrm{~g}$ in the scenario (Table 1). In women, the salt intake $(5.8 \mathrm{~g} /$ day) dropped to close to the recommended 5 grams. The intake of sucrose decreased moderately, from $47.1 \mathrm{~g}$ at baseline to $43.0 \mathrm{~g}$ $(8.7 \%)$ in the scenario (Table 1$)$.

Fiber intake increased from $21.2 \mathrm{~g} /$ day at baseline to $22.0 \mathrm{~g} /$ day in the scenario. It meant an increase of 3.9\% (Table 1).
Table 2 shows the mean nutrient intake by age group for men and women. All differences between the baseline and scenario in all age groups were statistically significant $(p<0.05)$ for men and women. In addition, testing the differences between the baseline and scenario among the age groups showed that for women, the differences varied across age groups for some nutrients. Especially the decrease in salt intake and increase in fiber intake were notably greater in the youngest age group. In men, the decrease in sugar intake and the increase in fiber intake seemed to be most prominent in the youngest age group, whereas the decrease in salt intake was smallest in this age group.

The mean nutrient intake differed significantly between the baseline and scenario in all education groups (Table 3). For men and women, the differences between the baseline and scenario differed significantly between education groups in the intake of hard fat and salt. The decreases were most prominent in men and women with the lowest education.

Table 2: Mean nutrient intake at baseline and in the scenario in men and women, by age.

\begin{tabular}{|c|c|c|c|c|c|c|c|c|c|c|c|c|}
\hline \multirow{3}{*}{ Men, $\mathrm{n}=585$} & \multicolumn{12}{|c|}{ Age group } \\
\hline & \multicolumn{3}{|c|}{$25-34, n=106$} & \multicolumn{3}{|c|}{$35-44, n=143$} & \multicolumn{3}{|c|}{$45-54, n=162$} & \multicolumn{3}{|c|}{$55-64, n=174$} \\
\hline & Baseline & Scenario & $\begin{array}{c}\text { Difference, } \\
\%\end{array}$ & Baseline & Scenario & $\begin{array}{c}\text { Difference, } \\
\%\end{array}$ & Baseline & Scenario & $\begin{array}{c}\text { Difference, } \\
\%\end{array}$ & Baseline & Scenario & $\begin{array}{c}\text { Difference, } \\
\%\end{array}$ \\
\hline Energy (kJ) & 10253.4 & 9591.1 & -6.5 & 9521.1 & 8957.9 & -5.9 & 9549.9 & 9014 & -5.6 & 8594.3 & 8108.7 & -5.7 \\
\hline Fat (g) & 101.6 & 84.3 & -17 & 93.2 & 78.5 & -15.7 & 93.3 & 78.7 & -15.7 & 85.8 & 71.5 & -16.6 \\
\hline SFA + Trans (g) & 41.6 & 26.5 & -36.4 & 37.4 & 24.9 & -33.4 & 37.2 & 24.3 & -34.7 & 34.4 & 21.5 & -37.6 \\
\hline Sugar (g) & 119 & 112.6 & -5.3 & 113.1 & 108 & -4.5 & 107.2 & 102.6 & -4.3 & 97.2 & 94 & -3.3 \\
\hline Fibre (g) & 19 & 20.3 & 6.4 & 21.4 & 22.3 & 4 & 22.7 & 24.1 & 5.5 & 23.5 & 24.2 & 3 \\
\hline Salt (g) & 9.5 & 8.5 & -10.1 & 8.7 & 7.8 & -11.3 & 9.3 & 8.2 & -11.6 & 8.3 & 7.4 & -11.6 \\
\hline Fibre (g/MJ) & 1.9 & 2.1 & 11.7 & 2.3 & 2.5 & 9.1 & 2.4 & 2.7 & 10.4 & 2.8 & 3.1 & 8.2 \\
\hline \multirow{3}{*}{ Women $n=710$} & \multicolumn{12}{|c|}{ Age group } \\
\hline & \multicolumn{3}{|c|}{$25-34, n=149$} & \multicolumn{3}{|c|}{$35-44, n=192$} & \multicolumn{3}{|c|}{$45-54, n=178$} & \multicolumn{3}{|c|}{$55-64, n=191$} \\
\hline & Baseline & Scenario & \begin{tabular}{|c|} 
Difference, \\
$\%$
\end{tabular} & Baseline & Scenario & $\begin{array}{c}\text { Difference, } \\
\%\end{array}$ & Baseline & Scenario & $\begin{array}{c}\text { Difference, } \\
\%\end{array}$ & Baseline & Scenario & $\begin{array}{c}\text { Difference, } \\
\%\end{array}$ \\
\hline Energy (kJ) & 7788.7 & 7360.4 & -5.5 & 7670.3 & 7232.6 & -5.7 & 7017.3 & 6659.6 & -5.1 & 6636.8 & 6322.7 & -4.7 \\
\hline Fat (g) & 78 & 66 & -15.4 & 75.2 & 62.8 & -16.5 & 67.3 & 56.8 & -15.5 & 62.9 & 53.6 & -14.7 \\
\hline SFA + Trans (g) & 31.8 & 21 & -33.9 & 30.4 & 20 & -34.3 & 27.3 & 18.3 & -33.1 & 24.3 & 16.3 & -32.9 \\
\hline Sugar (g)* & 102.1 & 97.1 & -4.9 & 95.1 & 89.4 & -5.9 & 89.9 & 86.3 & -4 & 83.2 & 80.1 & -3.7 \\
\hline Fiber $(\mathrm{g})^{*}$ & 19.5 & 20.5 & 5.3 & 20 & 20.6 & 2.9 & 20.7 & 21.4 & 3.3 & 21.8 & 22.3 & 2.1 \\
\hline Salt (g)* & 6.7 & 6 & -9.2 & 6.8 & 6 & -11 & 6.3 & 5.7 & -10.6 & 6.3 & 5.6 & -10.8 \\
\hline Fiber (g/MJ)* & 2.6 & 2.8 & 9.9 & 2.7 & 2.9 & 8.3 & 3.1 & 3.3 & 7.6 & 3.3 & 3.5 & 6.3 \\
\hline
\end{tabular}

All differences between the baseline and scenario were statistically significant $(\mathrm{p}<0.05)$

*The differences between the baseline and scenario were statistically significant $(p<0.05)$ among age group 
Table 3: Mean intake of energy and other dietary factors at baseline and in the scenario in men and women, by education

\begin{tabular}{|c|c|c|c|c|c|c|c|c|c|}
\hline \multirow{3}{*}{ Men, $n=585$} & \multicolumn{9}{|c|}{ Education } \\
\hline & \multicolumn{3}{|c|}{ Low, $n=187$} & \multicolumn{3}{|c|}{ Medium, n=191 } & \multicolumn{3}{|c|}{ High, n=200 } \\
\hline & Baseline & Scenario & Difference, $\%$ & Baseline & Scenario & Difference, \% & Baseline & Scenario & Difference, $\%$ \\
\hline Energy (kJ) & 9627.6 & 8998.3 & -6.5 & 9327.3 & 8790.2 & -5.8 & 9180.5 & 8689.1 & -5.4 \\
\hline Fat (g) & 95.9 & 78.8 & -17.8 & 89.6 & 74.8 & -16.5 & 92.2 & 78.7 & -14.6 \\
\hline $\mathrm{SFA}+\operatorname{trans}(\mathrm{g})^{*}$ & 40.1 & 24.7 & -38.5 & 36.6 & 23.8 & -34.9 & 35.4 & 23.7 & -33.1 \\
\hline Sugar (g) & 106.2 & 101.6 & -4.3 & 111 & 106.7 & -3.9 & 105.8 & 100.8 & -4.7 \\
\hline Fiber (g) & 22.6 & 23.6 & 4.1 & 21.8 & 22.9 & 5 & 20.9 & 22 & 4.9 \\
\hline Salt (g)* & 9.3 & 8.1 & -12.9 & 8.9 & 8 & -10.6 & 8.5 & 7.6 & -10.2 \\
\hline Fiber (g/MJ) & 2.4 & 2.7 & 9.7 & 2.4 & 2.7 & 10.2 & 2.4 & 2.6 & 9.6 \\
\hline \multirow{2}{*}{ Women, $n=710$} & \multicolumn{3}{|c|}{ Low, $n=235$} & \multicolumn{3}{|c|}{ Medium, $n=218$} & \multicolumn{3}{|c|}{ High, $n=252$} \\
\hline & Baseline & Scenario & Difference, \% & Baseline & Scenario & Difference, \% & Baseline & Scenario & Difference, \% \\
\hline Energy (kJ) & 7342.8 & 6909.6 & -5.9 & 7119.1 & 6749.8 & -5.2 & 7305.2 & 6960.3 & -4.7 \\
\hline Fat (g) & 73.1 & 61 & -16.7 & 68 & 57.5 & -15.5 & 70.2 & 60 & -14.6 \\
\hline $\mathrm{SFA}+\operatorname{trans}(\mathrm{g})^{*}$ & 30.5 & 19.6 & -35.7 & 26.8 & 18 & -33 & 27.5 & 18.8 & -31.7 \\
\hline Sugar (g) & 90.3 & 85.9 & -4.9 & 90.5 & 86.2 & -4.7 & 95.5 & 91.2 & -4.5 \\
\hline Fibre (g) & 19.7 & 20.5 & 3.7 & 20.9 & 21.6 & 3.1 & 21.1 & 21.7 & 2.9 \\
\hline Salt $(\mathrm{g})^{*}$ & 6.6 & 5.8 & -11.5 & 6.5 & 5.8 & -10 & 6.4 & 5.8 & -10 \\
\hline Fibre (g/MJ) & 2.8 & 3 & 8.6 & 3.1 & 3.3 & 7.6 & 2.9 & 3.2 & 7 \\
\hline
\end{tabular}

Contributors to nutrient intake

The potential effects of the scenario on nutrient content varied across food groups in both men and women (Table 4). Major sources of hard fat were fat spreads and cheeses, both at baseline and in the scenario. For men, sausages and cold cuts together were the second biggest contributor to total fat intake, whereas in women, cheeses remained a noteworthy contributor to fat intake.

Breads were the main contributors to salt intake at baseline, but as the salt content of breads was reduced remarkably in the scenario, the most important contributor to salt intake for men was sausages and cold cuts in the scenario. For women, breads remained the highest contributor to salt intake (Table 4).

Major sources of sucrose among the groups that were acknowledged in this study were biscuits and sweet pastries. The proportion of milk desserts (yoghurt, flavored curd, fermented milk and pudding) as a contributor to sucrose intake was reduced remarkably, to $66.0 \%$ in men and $61.1 \%$ in women (Table 4 ).

The major contributor to fiber intake both in men and women was bread, both at baseline and in the scenario (Table 4).

Table 4: Contributors to nutrient intake at baseline and in the scenario, in men and women

\begin{tabular}{|c|c|c|c|c|c|c|c|c|c|c|}
\hline \multirow{3}{*}{ Men, $n=585$} & \multicolumn{2}{|c|}{ Fat, g/d } & \multicolumn{2}{|c|}{ Hard fat, g/d } & \multicolumn{2}{|c|}{ Salt, g/d } & \multicolumn{2}{|c|}{ Sucrose, g/d } & \multicolumn{2}{|c|}{ Fibre, g/d } \\
\hline & & & & & & & & & & \\
\hline & Baseline & Scenario & Baseline & Scenario & Baseline & Scenario & Baseline & Scenario & Baseline & Scenario \\
\hline \multicolumn{11}{|l|}{ Food group } \\
\hline $\begin{array}{c}\text { Liquid milk } \\
\text { products (incl. } \\
\text { quark) }\end{array}$ & 4.1 & 0.8 & 3 & 0.5 & 0.4 & 0.4 & 0.5 & 0.51 & 0.1 & 0.1 \\
\hline Milk desserts & 2.3 & 0.5 & 1.6 & 0.3 & 0.1 & 0.09 & 4.9 & 1.7 & 0.3 & 0 \\
\hline Cheeses & 6.9 & 4.3 & 4.8 & 2 & 0.4 & 0.4 & 0 & 0 & 0 & 0 \\
\hline
\end{tabular}




\begin{tabular}{|c|c|c|c|c|c|c|c|c|c|c|}
\hline $\begin{array}{l}\text { Sausages and cold } \\
\text { cuts }\end{array}$ & 7.6 & 4.7 & 2.9 & 1.5 & 1.1 & 0.9 & 0 & 0 & 0.1 & 0 \\
\hline $\begin{array}{l}\text { Biscuits and sweet } \\
\text { pastries }\end{array}$ & 5.3 & 3.3 & 2.4 & 0.7 & 0.2 & 0.2 & 6 & 5.5 & 0.8 & 1.4 \\
\hline Breads & 2.8 & 2.7 & 0.5 & 0.4 & 1.4 & 0.8 & 0.9 & 0.7 & 9.8 & 10.5 \\
\hline Others & 46.1 & 45.8 & 14.4 & 14.2 & 4.9 & 4.9 & 38.9 & 38.8 & 10.9 & 10.9 \\
\hline Total & 92.5 & 77.5 & 37.2 & 24 & 8.9 & 7.9 & 51.2 & 47.2 & 22 & 23 \\
\hline \multicolumn{11}{|l|}{ Women, $n=710$} \\
\hline Fat spreads & 12.7 & 11.6 & 5.1 & 3.3 & 0.3 & 0.2 & 0 & 0 & 0 & 0 \\
\hline $\begin{array}{l}\text { Liquid milk } \\
\text { products (incl. } \\
\text { quark) }\end{array}$ & 2.4 & 0.6 & 1.7 & 0.4 & 0.3 & 0.3 & 0.4 & 0.5 & 0.1 & 0.1 \\
\hline Milk desserts & 3.2 & 0.6 & 2.2 & 0.4 & 0.1 & 0.1 & 4.8 & 1.9 & 0.3 & 0.1 \\
\hline Cheeses & 5.8 & 3.8 & 4.1 & 1.8 & 0.4 & 0.3 & 0 & 0 & 0 & 0 \\
\hline $\begin{array}{l}\text { Sausages and cold } \\
\text { cuts }\end{array}$ & 3.1 & 2.1 & 1.2 & 0.7 & 0.5 & 0.4 & 0 & 0 & 0 & 0 \\
\hline $\begin{array}{c}\text { Biscuits and sweet } \\
\text { pastries }\end{array}$ & 4.8 & 2.9 & 2.1 & 0.6 & 0.2 & 0.2 & 5.8 & 4.8 & 0.7 & 1.3 \\
\hline Breads & 2.5 & 2.1 & 0.5 & 0.3 & 1.1 & 0.6 & 0.7 & 0.5 & 7.7 & 8.1 \\
\hline Others & 36.1 & 35.8 & 11.4 & 11.2 & 3.7 & 3.7 & 31.9 & 31.9 & 11.8 & 11.8 \\
\hline Total & 70.5 & 59.5 & 28.3 & 18.8 & 6.5 & 5.8 & 43.6 & 39.6 & 20.5 & 21.2 \\
\hline
\end{tabular}

\section{Discussion}

In this study, we estimated the impact of replacing ordinary food products with the Heart Symbol compliant foods on nutrient intake in the Finnish working-age population. A scenario was created and compared to a baseline situation based on the National FINDIET 2012 Survey [13]. In general, after replacing non-compliant products with Heart Symbol compliant foods, the nutrient intake distributions based on the National FINDIET 2012 Survey shifted closer to meeting the nutrition recommendations. The results of this study showed a significant decrease in fat, hard fat and salt intake.

Intake of hard fat reduced significantly in all age and educational groups, the mean intake being less than recommended $10 \mathrm{E} \%$ in the scenario. Major food groups responsible for this reduction were dairy products, fat spreads, and biscuits and sweet pastries. Total fat intake was also reduced after replacement in the scenario, mainly due to changes in the food composition of dairy products.

Intake of salt was remarkably reduced for all age and educational groups. It is worth noticing that the impact appeared to be largest among men and women with the lowest educational level. Salt intake in Finland has decreased due to successful salt reduction strategies, but the intake is still above the recommended $[13,19]$. Hendriksen et al. [20] have estimated in their modeling study that reducing salt intake by $30 \%$ would reduce the prevalence of stroke by $6.4 \%$ in Finland. To achieve this goal, additional salt reductions are required Martikainen et al. [21] modeled that the burden of cardiovascular diseases can be reduced through even modest reductions in salt intake. Reducing salt by $1 \mathrm{~g}$ per day in Finnish adults (aged 30-74 years) was estimated to lower systolic blood pressure by $1.185 \mathrm{mmHg}$ among hypertensive subjects and by $0.595 \mathrm{mmHg}$ among subjects with normal blood pressure. These changes together with alterations in the dietary quality of fat and its impact on serum cholesterol levels could potentially diminish cardiovascular disease cases by 8000-13 000 during the period 2010-2030.

Intake of fiber was increased and sugar was decreased in all age and educational groups, but the effects on intake were modest. However, beverages and sweets, which are the main contributors to sucrose intake, were not included in the modelling study. When it comes to fiber intake, bread is the most important source of fiber in Finland, being rather fiber-rich to begin with.

Recent studies by Temme et al. [6] and Schickenberg et al. [7] showed similar results. Temme and colleagues evaluated the effects of replacing foods according to health logo criteria on the intake of saturated fatty acids, sugar and sodium in young Dutch adults [6]. They found that in the most optimal replacement scenario, where all the replaceable foods were substituted with foods complying with the health logo criteria, the intake reductions in saturated fatty acids, sodium and sugar were considerable. Schickenberg et al [7] replaced only a few products high in saturated fat with alternatives lower in saturated fat and concluded that it significantly reduced saturated fat intake, while the proportion of individuals meeting the dietary recommendations increased.

Roodenburg et al. [22] evaluated the potential effect of the nutrient profiling system called the Choices Programme on nutrient intake in young Dutch adults. In a later study they fur- 
ther analyzed their previous work [8] and expanded the analysis to cover a broader set of nutrients. Their modelling study showed that diets containing foods complying with the health logo criteria shifted the nutrient intake closer to meeting the recommendations. In their former study, Roodenburg et al. [8] found that replacing all the normally consumed foods with foods that are eligible to carry a Choices logo showed substantial improvements in the intake of saturated fatty acids (SFA), total sugar and fiber. In their study, all the foods were replaced unless it was impossible to find replacement food, as opposed to our study, where only foods in defined food groups were replaced.

A Brazilian study showed that replacing typical products by products complying with the Choices criteria in the Brazilian population reduced the intake of saturated fat by $52 \%$ and trans fatty acids by $92 \%$. Sodium intake was reduced by $47 \%$, whereas fiber intake was increased by $87 \%$ [9].

This study does have some limitations. Our data are not a representative sample of the total number of products bearing a Heart Symbol logo, as only defined food groups were included in the study. In addition, foods eaten as such were included in the scenario, not those consumed as ingredients in dishes. However we chose food groups that were assumed to have great potential to reduce the intake of especially hard fat and sodium. Future research should try to expand the modelling so that different types of foods, e.g. ready-to-eat meals, main dishes and take-away foods could be included in the scenarios.

It should be kept in mind that the study shows the potential of using products labeled with the Heart Symbol. Previous studies have shown that the label is well-known (total familiarity $85 \%$ ) in Finland and the reported use of products has increased [23]. Nearly $60 \%$ of Finnish men and almost three quarters (73\%) of women aged 15-64 years have reported using products labeled with the symbol [23]. However, actual use of the products is unknown and people may not use these products regularly and may not utilize a whole assortment of products in different food groups. Product acceptability is an important aspect to consider when applying the results of the study to real life. Supposed differences in taste between products may be an obstacle to some consumers.

There were many assumptions made in this simulation, for example, regarding the theoretical replacing of foods. However, the replacements were discussed with four nutritionists. In addition, the food groups of the Heart Symbol and food categories in the Finnish National Database were not completely comparable. People were also assumed to consume the same amount of replacement foods as their original choice, which may be underestimated, as people may eat more if they perceive the food to be healthier. Level of energy intake was reduced after the replacements but energy corrections were not conducted. It might be preferable to minimize the possible compensatory behavior by choosing products with comparable energy content.

Originally, we created two scenarios to test potential effects of the Heart Symbol. In another scenario (data not shown), products not complying with the Heart Symbol criteria were replaced by similar foods complying with the maximum (minimum in fiber) levels of the Heart Symbol criteria, i.e. using the criteria used to award the symbol. However, since the results were almost identical, the findings were presented only for the scenario, in which non-compliant foods we replaced by using the average nutritional content of products labeled with the Heart Symbol on the Finnish market. Where ever minor differences were found between these two scenarios, the reductions in the presented scenario were bigger than in another scenario suggesting that products labeled with the Heart Symbol on the market have nutrient contents that are even better than the criteria used to award the symbol.

Despite the limitations, this study provides an insight into the potential effects of a front-of-package label on nutrient intake. Dietary data for the study were collected using sophisticated dietary assessment methods by trained nutritionists, and nutrient intake was calculated with software based on the Finnish Food Composition Database Fineli ${ }^{\circledR}$. Nutrient intake data were derived from a large national population study that is representative of Finnish working-aged adults. The results can be generalized to some extent to various areas, age groups and educational groups in Finland.

Our findings suggest that there is a great potential to reduce the intake of hard fat and sodium by using products bearing the Heart Symbol. Given that modelling was carried out using only four main food groups, the overall potential for improving nutrient intake could even be greater. The development of healthier products and increased availability of products with improved nutrient content would enhance the diets of Finnish adult population.

\section{Acknowledgment \\ Financial support}

This research was funded by the Nordic Council of Min-

isters

\section{Authorship}

All authors contributed to the formulation of the research questions and study design and interpretation of the empirical results. KA-K wrote the first draft, SR finalized the drafts and all authors commented on the manuscript. HT carried out the statistical analyses and planned them together with KA-K, SR, ML-K, LT and SMV. HT and SMV coordinated the data collection in 2012.

\section{Ethical Standards}

This study was conducted according to the guidelines laid down in the Declaration of Helsinki (7th Revision, 2013). The Coordinating Ethics Committee of Helsinki and Uusimaa Hospital District (HUS) approved the FINRISK 2012 protocols. All participants received information about the study and provided their written informed consent.

\section{References}

1. WHO. Diet, Nutrition and the Prevention of Chronic Diseases.World Health Organization Technical Report Series. 2003;916:i-viii,1-149.

2. WHO. Global Strategy on Diet, Physical Activity and Health. Geneva: World Health Organization.2004.

3. Vyth EL, IH Steenhuis, AJ Roodenburg, J Brug, JC Seidell. Front-of-Pack Nutrition Label Stimulates Healthier Product Development: A Quantitative Analysis. The International Journal of Behavioral Nutrition and Physical Activity. 2010;7:65. doi: 10.1186/1479-5868-7-65.

4. Young L, B Swinburn. Impact of the Pick the Tick Food Information Programme on the Salt Content of Food in New Zealand. Health Promotion International. 2002;17(1):13-19. 
5. Williams, Peter, Anne McMahon, and Rebecca Boustead. A Case Study of Sodium Reduction in Breakfast Cereals and the Impact of the Pick the Tick Food Information Program in Australia. Health Promotion International. 2003;18(1):51-56.

6. Temme EH, H van der Voet, AJ Roodenburg, A Bulder, G van Donkersgoed, J van Klaveren. Impact of Foods with Health Logo on Saturated Fat, Sodium and Sugar Intake of Young Dutch Adults. Public Health Nutrition. 2011;14 (4):635-644.

7. Schickenberg B, P van Assema, J Brug, J Verkaik-Kloosterman, MC Ocke, NK de Vries. Replacing Foods High in Saturated Fat by Low-Saturated Fat Alternatives: A Computer Simulation of the Potential Effects on Reduction of Saturated Fat Consumption. The British Journal of Nutrition. 2009;102(3):478-483. doi: 10.1017/S0007114508190298.

8. Roodenburg AJC, EHM Temme, O Howell Davies. Potential Impact of the Choices Programme on Nutrient Intakes in the Dutch Population. Nutrition Bulletin. 2009;34(3):318-323.

9. De Menezes EW, V Lopes Tdo, ER Mazzini, MC Dan, C Godoy, and E. B. Giuntini. Application of Choices Criteria in Brazil: Impact on Nutrient Intake and Adequacy of Food Products in Relation to Compounds Associated to the Risk of Non-Transmissible Chronic Diseases. Food Chemistry. 2013;140 (3):547-552. doi: 10.1016/j.foodchem.2013.02.031

10. Lahti-Koski M, S Helakorpi, M Olli, E Vartiainen and P Puska. Awareness and use of the Heart Symbol by Finnish Consumers. Public Health Nutrition. 2012;15(3):476-482. doi: 10.1017/S136898001100187X

11.National Nutrition Council. Health from Food! Finnish Nutrition Recommendations 2014 [in Finnish]. Helsinki: Juvenes OY. 2014.

12. Kinnunen, Tarja I. The Heart Symbol: A New Food Labelling System in Finland. Nutrition Bulletin. 2000;25(4):335-339. DOI: 10.1046/j.1467-3010.2000.00079.x

13. Helldán A, S Raulio, M Kosola, H Tapanainen, M-L Ovaskainen, and $S$ Virtanen. Reports of the National Institute for Health and Welfare (THL) 16/2013. The National FINDIET 2012 Survey. Helsinki.

14. Jousilahti, Pekka, Tiina Laatikainen, Markku Peltonen, Katja Borodulin, Satu Männistö, Antti Jula, et al. Primary Prevention and Risk Factor Reduction in Coronary Heart Disease Mortality among Working Aged
Men and Women in Eastern Finland Over 40 Years: Population Based Observational Study. BMJ. 2016;352:i721. doi:10.1136/bmj.i721.

15.L Saarikoski, L Lund, A Juolevi, M Grönholm, A Helldán, M Peltonen, et al. Reports of the National Institute for Health and Welfare (THL) 22/2013. The National. FINRISK 2012 Study [in Finnish]. Helsinki: THL.

16. Männistö, Satu, Marja-Leena Ovaskainen, and Liisa Valsta. Publications of the National Public Health Institute (KTL) B3/2003. The National FINDIET 2002 Study. Helsinki: Publications of the National Public Health Institute (KTL) B3/2003.

17. Reinivuo H, T Hirvonen, ML Ovaskainen, T Korhonen, LM Valsta. Dietary Survey Methodology of FINDIET 2007 with a Risk Assessment Perspective. Public Health Nutrition. 2010;13(6A):915-919.

18. National Institute for Health and Welfare. Fineli - Finnish Food Composition Database. Release 15. National Institute for Health and Welfare. 2012.

19. Reinivuo H, LM Valsta, T Laatikainen, J Tuomilehto, P Pietinen. Sodium in the Finnish Diet: II Trends in Dietary Sodium Intake and Comparison between Intake and 24-H Excretion of Sodium. European Journal of Clinical Nutrition. 2006;60(10):1160-1167.

20. Hendriksen MA, JM van Raaij, JM Geleijnse, J Breda and HC Boshuizen. Health Gain by Salt Reduction in Europe: A Modelling Study. PloS One. 2015;10(3):e0118873. doi:10.1371/journal.pone.0118873

21. Martikainen JA, EJ Soini, DE Laaksonen, L Niskanen. Health Economic Consequences of Reducing Salt Intake and Replacing Saturated Fat with Polyunsaturated Fat in the Adult Finnish Population: Estimates Based on the FINRISK and FINDIET Studies. European Journal of Clinical Nutrition. 2011;65(10):1148-1155. doi: 10.1038/ejcn.2011.78.

22. Roodenburg AJ, AJ van Ballegooijen, $M$ Dotsch-Klerk, $H$ van der Voet, JC Seidell. Modelling of Usual Nutrient Intakes: Potential Impact of the Choices Programme on Nutrient Intakes in Young Dutch Adults. PloS One. 2013;8(8):e72378.

23. Helldán A, S Helakorpi, S Virtanen, A Uutela. Health Behaviour and Health among the Finnish Adult Population, Spring 2013. Helsinki: National Institute for Health and Welfare (THL). 2013. Report 21/2013 